\title{
Cross Pollinating Minds for the Future
}

A few years ago while doing research for a diabetes program it became clear that the statistics were all but abysmal worldwide. By 2035 , entire nations were expected to be bankrupt as a result of healthcare costs for diabetes and its comorbid conditions obesity and cardiovascular disease. Healthcare expenses and productivity loss needed to be cauterized.

Not all the research was negative, however. I found studies of telephonic health support where mobile technology helped improve medical conditions. One was from Kaiser Permamente in Northern California, where $73 \%$ of patients reported that coaching helped achieve their weight goal. Another from the UN Foundation and Vodafone Foundation Partnership reported that patients with tuberculosis in Thailand increased drug adherence $90 \%$. In Kenya, text messages increased HIV drug intake $12 \%$.

With the acceleration of digital health and mhealth, healthcare has entered a new frontier. To help meet this need, we present our premier issue of Telehealth and Medicine Today ${ }^{\mathrm{TM}}$, a scholarly, CME-accredited open-access e-journal. With it, we strive to dispatch answers to key issues in telemedicine through publishing datadriven research and commentary, complimented by case studies and real world evidence, from across the United States and around the globe.

Our editorial will address the needs reported in the 2010 WHO report on Telemedicine Opportunities \& Developments in Member States. In it, almost $70 \%$ of responding countries desired further information on the cost and cost-effectiveness of telemedicine solutions, while over $50 \%$ of countries wanted more information on the infrastructure necessary to implement telemedicine solutions. Information on the clinical uses of telemedicine was the second-most cited information need-by almost $60 \%$ of responding countries.

Telehealth and Medicine Today ${ }^{\mathrm{TM}}$ promises a most rapid peer review process-more rapid than any other journal in our market. Our commitment is to publish high-quality manuscripts within one month in most cases. Authors will hear from us within the week of submission. Our board is hands-on and the approach is team oriented both in-house and with authors. This will speed citations, referencing, and importantly, disseminate cutting edge research and insights. We want to cross-pollinate and challenge minds with timely publication of what is current and what is possible in future.

On behalf of the board and the entire operations team, we are proud to be part of the telemedicine solution. Thank you for your support as readers, advertisers, and partners on this journey. We welcome your feedback and encourage your contributions.

Tory Cenaj

Founder and Publisher: Telehealth and Medicine Today ${ }^{\mathrm{TM}}$ 